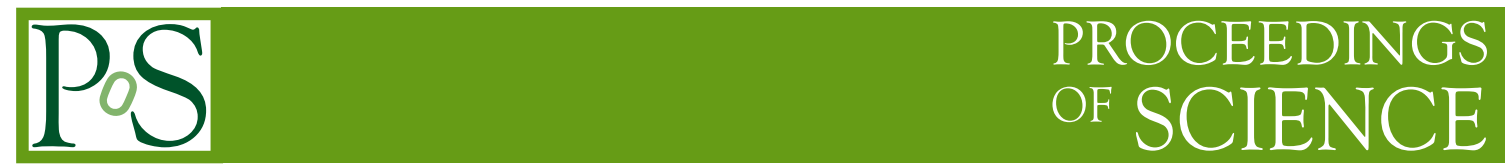

\title{
Commissioning the CMS trigger with cosmic rays
}

\section{Tuula MAKI*†}

\section{CERN}

E-mail: tuula.maki@cern.ch

The CMS experiment has collected about 300 million cosmic ray events. This has provided an excellent opportunity to test the trigger and prepare for the LHC collision data. This paper describes some of the L1 trigger studies we performed with these events.

European Physical Society Europhysics Conference on High Energy Physics July 16-22, 2009

Krakow, Poland

*Speaker.

${ }^{\dagger}$ for the CMS Collaboration 


\section{Introduction}

The CMS experiment recorded about 300 million events in October-November 2008 in an exercise called CRAFT (Cosmic Run At Four Tesla). These events were either coming from cosmic rays or from detector noise.

The first level (L1) of the CMS trigger is made of custom-made hardware processors. It selects the most interesting events using information from the three muon detectors (drift tube chambers (DT), cathode strip chambers (CSC) and resistive plate chambers (RPC)) and from the electromagnetic (ECAL) and hadronic (HCAL) calorimeters, and reduces the rate to $100 \mathrm{kHz}$. The rate is further decreased by the High Level Trigger (HLT) processor farm to $100 \mathrm{~Hz}$ [1].

Cosmic ray muons arrive from many directions, they are not timed with the LHC-clock, and they do not necessarily cross the interaction point. Therefore, configuration of the muon triggers was relaxed to give high efficiency for this kind of muons. The calorimeter triggers were operated in the standard LHC way.

Only the simplest trigger algorithms were activated in the Global Trigger: any muon candidate from any sub-detector with any $p_{T}$, single electron/photon $(\mathrm{e} / \gamma)$ candidate with $E_{T}>1 \mathrm{GeV}$, or single jet candidate with $E_{T}>10 \mathrm{GeV}$. Their measured trigger rates were $300 \mathrm{~Hz}, 23 \mathrm{~Hz}$ and $140 \mathrm{~Hz}$, respectively.

A detailed description of cosmic ray studies with the CMS L1 trigger can be found in [2].

\section{Synchronization}
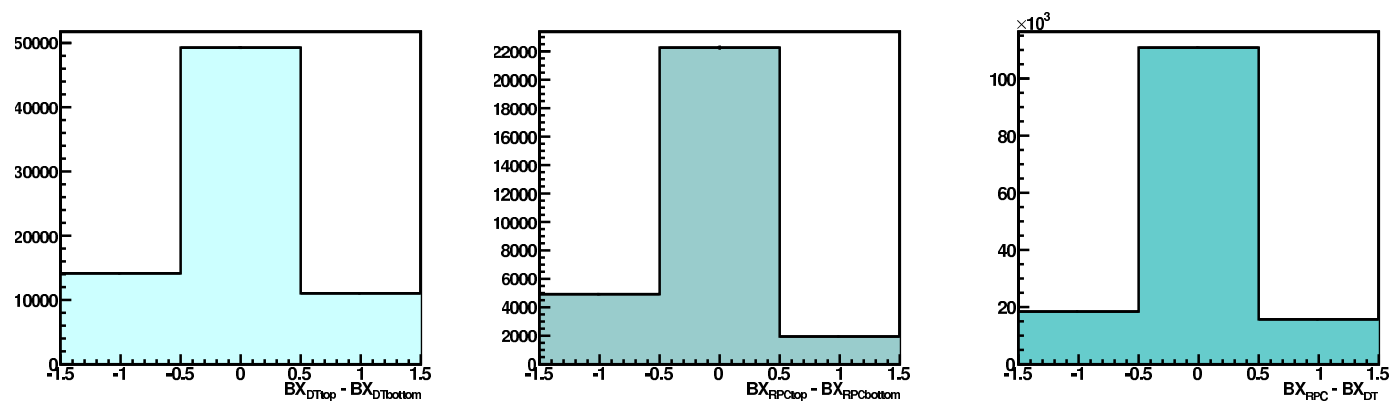

Figure 1: Synchronization of muon triggers. The plots show the time difference in bunch crossings between the L1 muon candidates from top and bottom parts of the DT, from the top and bottom parts of the RPC and from the RPC and DT. The candidates are created by the same cosmic ray muon.

Cosmic ray muons mainly cross the detector from the top to bottom. This was taken into account in the muon trigger synchronization, i.e. the trigger signal from the top part of the detector was delayed by the average flight time from top to the bottom part of the detector.

The synchronization with cosmic rays is only possible to a limited extent. It was checked by calculating the time difference in terms of bunch crossings between signals from various trigger components (Figure 1). For calorimeter triggers, the trigger signals were adjusted to provide the highest coincidence rate above the noise level. The majority of the signals are synchronized 


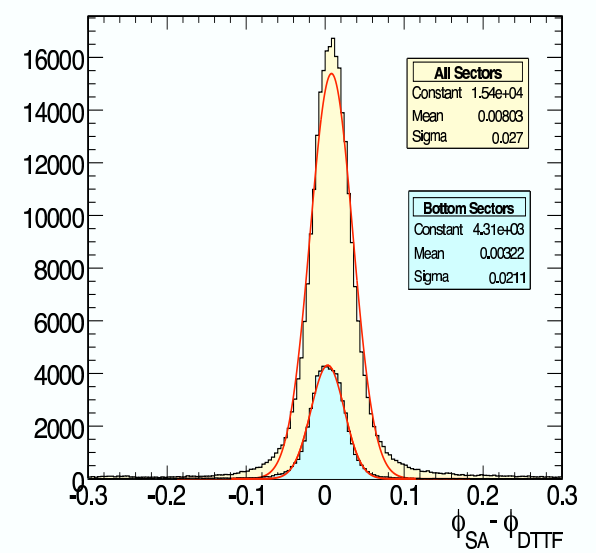

Figure 2: DT muon candidate $\phi$ resolution. The yellow histogram shows the $\phi$ difference obtained from all the sectors, and the blue histogram shows the difference for bottom sectors only. SA stands for a standalone muon.

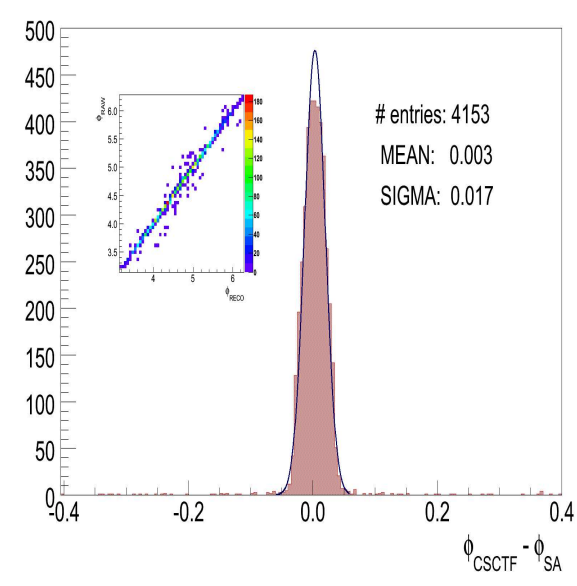

Figure 3: CSC muon candidate $\phi$ resolution. The $\phi$ difference is shown for bottom sectors only. SA stands for a standalone muon.

\section{Emulator studies}

We have developed a software which emulates the L1 trigger bit by bit. This tool can be used to validate the trigger performance on event by event basis, and to simulate the trigger response in Monte Carlo simulation. The emulator was run for all the trigger sub-systems and global triggers with a sub-set of CRAFT events. The agreement between the emulator and real triggers is between 95-100\%, depending on the trigger subsystem. The disagreements were either expected due to some cosmic-ray-specific configurations not precisely implemented in the software or identified as firmware mistakes and fixed.

\section{Resolutions}

In the resolution studies L1 trigger coordinates were compared to offline quantities. The DT track finder $\phi$ and $\eta$ coordinates were compared to offline standalone muons (only muon detector is used). The $\phi$ resolution is shown in Figure 2, and it is compatible with expectations. The RPC $\phi$ resolution is also as expected.

The CSC track finder candidates were compared to reconstructed muons which were selected to pass close to the interaction point. Their resolution is similar to the DT track finder resolutions as shown in Figure 3. Resolution studies were also performed for e $\gamma$ triggers. The $\phi$ and $\eta$ resolutions are consistent with the granularity of the e/ $\gamma$ triggers, and the $E_{T}$ resolution distribution is consistent with expectations.

\section{Efficiencies}

The DT trigger efficiency study uses muons which traverse from top to bottom. The bottom part of the detector is required to have a matching pair of a trigger candidate and a reconstructed 


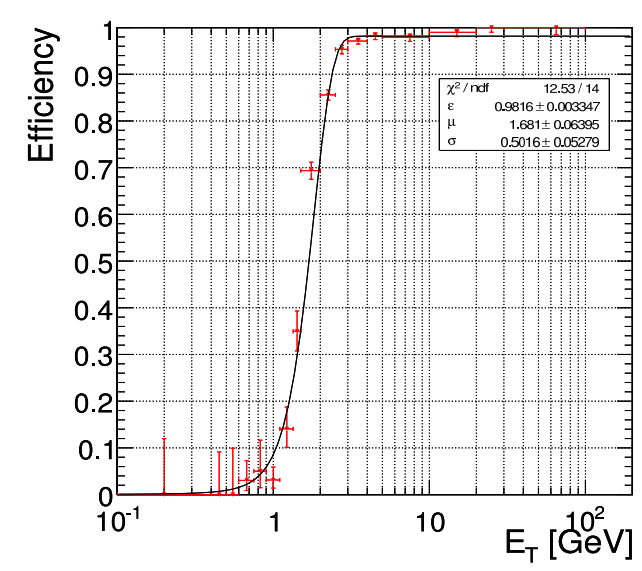

Figure 4: $\mathrm{L} 1 \mathrm{e} / \gamma$ trigger candidate efficiency as a function of $E_{T}$.

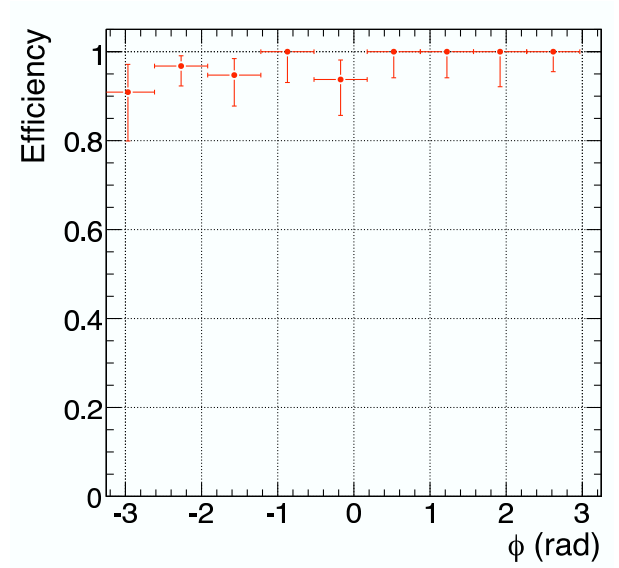

Figure 5: L1 single jet trigger candidate efficiency as a function of $\phi$.

standalone track. The track is propagated to the top part of the detector, and a trigger candidate is looked for. The efficiency in the active parts of the detector is above $95 \%$ which is compatible with the efficiency of individual chambers for cosmic muons. A similar study was performed for the RPC trigger, and the measured efficiency is between 85 and $90 \%$ in the central $p_{T}$ region. The CSC efficiency study uses triggers in one endcap and offline tracks from the central tracker to measure the efficiency of the trigger on the other endcap. The study yields an efficiency of 97-100\%.

The efficiency of the e $/ \gamma$ trigger was studied at the level of trigger primitive generation and at the level of building of the $\mathrm{L} 1 \mathrm{e} / \gamma$ candidate. Muon triggered events with muon pointing superclusters were used as the base of the analysis. Figure 4 shows the e/ $\gamma$ candidate efficiency as a function of the $E_{T}$. The difference between the turn-on point and the trigger threshold, $1 \mathrm{GeV}$, is a combination of known factors that are different for the L1 $E_{T}$ and the reconstructed $E_{T}$.

The e/ $\gamma$ triggered sample was used to study the efficiency of the jet triggers. An event was considered efficient if a L1 jet candidate was found close to an offline jet. The efficiency as a function of $\phi$ is shown in Figure 5.

\section{Summary}

Our experience from cosmic ray runs has proved that the CMS L1 trigger runs stably and reliably. We performed emulator studies, synchronized the triggers, studied resolutions between trigger and offline quantities and measured trigger efficiencies. From the studies, we conclude that the muon and e/ $\gamma$ triggers are commissioned and well understood. The jet and energy sum triggers remain to be commissioned during cosmic ray runs scheduled before the first LHC beam.

\section{References}

[1] CMS Collaboration, “The CMS experiment at the CERN LHC”, JINST 0803 (2008) S08004.

[2] CMS Collaboration, "Performance of the CMS Level-1 Trigger during Commissioning with Cosmic Rays", to be published. 